\title{
A Very Rare Case Of Primary Renal Hydatidosis
}

\author{
Dr. Ashis Kumar Saha ${ }^{1}$, Dr. Tapas Kumar Majhi ${ }^{1}$, Dr. Chhandas Das ${ }^{2}$, Dr. \\ Madhumita Mukhopadhyay ${ }^{2}$ \\ ${ }^{I}$ Departmen of Surgery, Bankura Sammilani Medical College, Bankura, India \\ ${ }^{2}$ Department of Pathology, IPGME\&R, Kolkata, India
}

\begin{abstract}
Primary Kidney involvement in echinococcosis is extremely rare. We present a rare case of primary left renal hydatid with gross hydatiduria with history of short haematuria. We diagnosed the case from the typical clinical history of intermittent passage of small, white, balloon-like, grape-sized structures in the urine for the last one month. On radiological imaging, USG abdomen revealed multiseptate cyst in the left kidney; liver was normal. The CT scan revealed a cystic lesion in the left kidney. Left nephroureterectomy was done by flank extraperitoneal approach and diagnosis was confirmed post operatively by the gross appearance and microscopy.
\end{abstract}

Keywords - Echinococcosis, Hydatiduria, Nephroureterectomy.

\section{INTRODUCTION}

Kidney involvement in echinococcosis is extremely rare, constituting only 2-3\% of all cases. Primary involvement of the kidney without the involvement of the liver and lungs is even rarer. Hydatiduria accompanies only $10-20 \%$ of all cases of renal hydatidosis and is usually microscopic. We present a rare case of primary left renal hydatid with gross hydatiduria. The diagnosis of primary hydatid cyst of the kidney, in the absence of hydatiduria, is usually radiological as most patients have negative immunological tests. We also present the salient radiological features of primary hydatid cyst of kidney.

\section{CASE REPORT}

A 25-year-old male presented with history of intermittent passage of small, white, balloon-like, grape-sized structures in the urine for the last one month. He also had intermittent haematuria for 15 days. Abdominal examination did not reveal any palpable lump. Rest of the systemic examination was normal.

His routine blood investigations were normal with no eosinophilia and normal renal function tests. Xray chest P-A view was normal. The USG abdomen revealed multiseptate cyst in the left kidney; liver was normal. The $\mathrm{CT}$ scan revealed a cystic lesion in the left kidney.

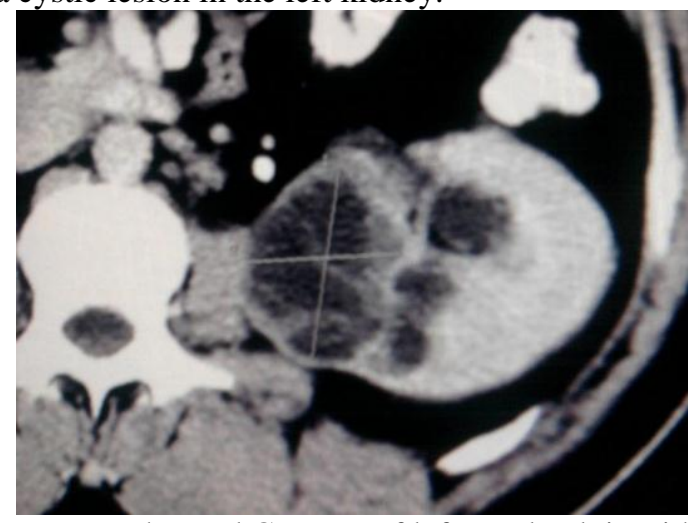

Figure 1: showing contrast enhanced CT scan of left renal pelvis with a multisepted cyst.

Patient was planned for surgery by flank extraperitoneal approach. Left nephroureterectomy was done. Patient received four weeks of preoperative Albendazole which was continued for four weeks postoperatively. The resected specimen showed kidney with bag of cysts with multiple daughter cysts. The histopathological examination was consistent with right renal hydatid disease and multiple daughter cysts. 


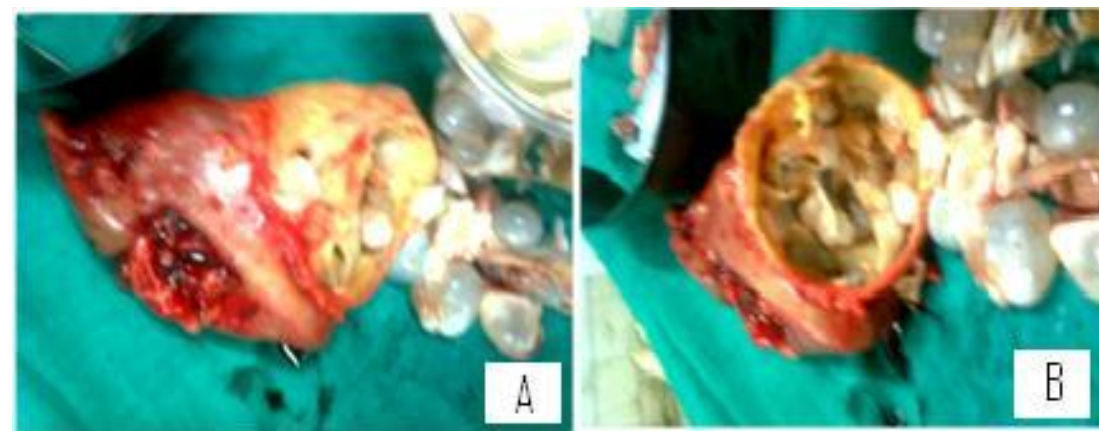

Figure 2: [A] showing the resected left kidney with sprouting grape like cysts, [B] showing inside of renal pelvis

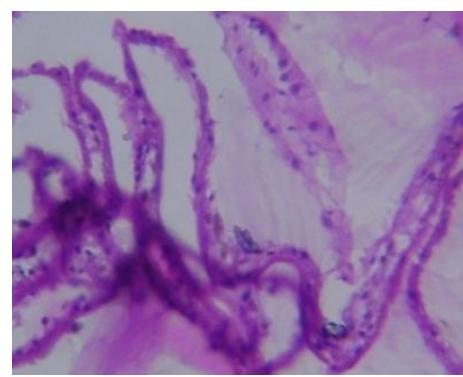

Figure 3: showing hydatid cyst in MGG stain (x10000)

\section{DISCUSSION}

Echinococcosis is a worldwide zoonosis produced by the larval stage of the Echinococcus tapeworm. In this case, hydatid disease was caused by one of the main types, E granulosus. The adult worm lives in the proximal small bowel of the definitive host, attached by hooklets to the mucosa. Eggs are released into the host's intestine and excreted in the feces. Humans may become intermediate hosts through contact with a definitive host (usually a domesticated dog) or ingestion of contaminated water or vegetables. The ovum loses its protective layer as it is digested in the duodenum. Once the parasitic embryo passes through the intestinal wall to reach the portal venous system or lymphatic system, the liver acts as the first line of defense and is therefore the most frequently involved organ. In humans, hydatid disease involves the liver in approximately $75 \%$ of cases and the lung in $15 \%$. Secondary involvement due to haematogenous dissemination may be seen in almost any anatomic location. Kidney involvement in echinococcosis is extremely rare (2\%-3\% of cases), even in areas where hydatid disease is endemic. Renal hydatid cysts usually remain asymptomatic for many years. There are no pathognomonic clinical signs except cystic rupture into the collecting system, which leads to acute renal colic and hydatiduria ${ }^{[1,2]}$. It is not clear how the hydatid embryo reaches the kidney in cases of primary hydatid disease but it is postulated that it must pass through the portal system into the liver and retroperitoneal lymphatics. The hydatid cyst of the kidney is considered closed if all three layers of the cyst i.e. pericyst, ectocyst and endocyst are intact. When the cyst is no longer protected by the third layer i.e. pericyst or by the lining of collecting system it is considered to be an exposed cyst. If all the three layers of the cyst have ruptured resulting in free communication with the calyces and pelvis, it is called an open or communicating cyst. Cystic rupture into the collecting system, causing hydatiduria is pathognomonic, though seen in only $10-20 \%$ of renal hydatidosis and is usually microscopic. Gross passage is rather uncommon, but has a tremendous diagnostic utility. Since the cysts passed in the urine are daughter cysts they lack the third layer pericyst, which is contributed by the host around the mother cyst, as was in this case. Eosinophilia is noted in about $50 \%$ cases. Serological tests in primary renal hydatidosis are usually negative. Advanced radiological techniques like CT scan and MRI remain the mainstay of diagnosis.

Plain films are usually nonspecific and mostly nonrevealing. A thin rim of calcification delineating a cyst is suggestive of an echinococcal cyst. Ultrasonography helps in the diagnosis of hydatid cysts when the daughter cysts and hydatid sand are demonstrated. On changing the patient's posture under real time, there is shifting of hydatid sand, which may give rise to the "falling snowflake pattern". The accuracy of ultrasound evaluation remains operator-dependent. The CT scan has an accuracy of $98 \%$ and sensitivity to demonstrate the daughter cysts. The CT scan usually demonstrates an expansile, hypo-attenuating tumor with a well-defined wall and daughter cysts within the parent $\mathrm{cyst}^{[3]}$. Magnetic resonance imaging usually reveals a solitary, highsignal-intensity mass consisting of multiple thin-walled lesions and outlined by a thick, hypointense rim. The high signal intensity is due to the characteristic high fluid content of the mass. The small peripheral cysts are 
usually hypointense relative to the central component. The MRI shows the cysts adequately, but MRI offers no real advantage over $\mathrm{CTscan}^{[4]}$.

In general, surgery is the treatment of choice in renal hydatid cyst. Kidney-sparing surgery (removal of hydatid cyst with pericystectomy) is possible in most cases (75\%). Nephrectomy (25\% of cases) must be reserved for destroyed kidney ${ }^{[5]}$. Very few cases of laparoscopic removal of renal hydatid are reported. There is fear of cyst rupture and dissemination during dissection, entrapment and removal of the hydatid cyst during laparoscopy. Utmost care should be taken during the surgery to prevent spillage and resultant disseminated hydatidosis. Pre and postoperative one-month courses of Albendazole should be considered in order to sterilize the cyst, decrease the chance of anaphylaxis and decrease the tension in the cyst wall (thus reducing the risk of spillage during surgery) and to reduce the recurrence rate postoperatively. During kidney-sparing surgery scolicidal solutions such as hypertonic saline should be used before opening the cavities to kill the daughter cysts and therefore prevent further spread or anaphylactic action.

\section{REFERENCES:}

[1] Saxena S, Gupta R, Nigam DK, Tahiliani ND, Saxena KN. Hydatid cyst of kidney presenting as hydatiduria. J Assoc Physicians India. 1990;38:359-60.

[2] Unsal A, Cimentepe E, Dilmen G, Yenidunya S, Saglam R. An unusual cause of renal colic: Hydatiduria. Int J Urol 2001;8:319-21

[3] Pedrosa I, Saiz A, Arrazola L, Ferreiros J, Pedrosa CS. Hydatid disease: Radiologic and pathologic features and complications. Radiographics 2000;20:795-817.

[4] Volders WK, Gelin G, Stessens RC. Hydatid cyst of the kidney: Radiologic-pathologic correlation. Radiographics 2001;21:S255-60

[5] Von Sinner WN, Hellstrom M, Kagevi I, Norlen BJ. Hydatid disease of the urinary tract. J Urology 1993;149:577-80. 